\title{
Enrico Biancardi, Leonard W. Panella and Robert T. Lewellen: Beta maritima: The Origin of Beets
}

\author{
Springer, New York, 2012, 293 pp, ISBN: 978-1-4614-0841-3
}

\section{Piergiorgio Stevanato}

Published online: 5 July 2013

(C) Society for Sugar Research \& Promotion 2013

To the best of my knowledge, this book is the first devoted entirely to sea beet (Beta maritima L.), the wild ancestor of all cultivated beets. The wild beets have always been harvested both for food and as a medicinal herb. Due to the latter use, references are frequently in old or obscure books and difficult to find and cite. The wild species gave rise to different cultivated offspring employed firstly as vegetable, later as fodder. At the end of 1700 s, the most important purpose was found, i.e., the extraction of sugar.

The book was written by leading specialists in the crop and collects and summarizes numerous references published on B. maritima and its cultivated offspring Beta vulgaris L. Particularly useful are chapters one, which describes the story of the wild species, and four on taxonomy. Chapter 1 includes biographies, results, and collaborations among internationally known scientists and breeders who were pivotal in sea beet research and collection, and who isolated and improved cultivated germplasm with genetic traits extracted from the wild plant. Chapter 6 describes the traits transferred to sugar beet, mainly genetic resistance against pests and diseases. The crop wild species crosses easily with cultivated beets. This facilitates the transmission of genetic traits seemingly lost during domestication and crop improvement. Because the authors of the book were or are directly involved in breeding activities, some previously unpublished information and historical insights are included, such as the section regarding the resistance to rhizomania and to cercospora leaf spot. The color illustrations (some unedited or original) are among the most valuable part of the book. The appendices are original and useful, i.e., the English translation of the numerous Latin names given to B. maritima.

The book is recommended because currently there is much interest in wild beets as a source of new genetic variability for pests and diseases resistance. In addition, $B$. maritima has become a model cross pollinated plant for investigating gene flow in other crops that involve the use of GMs.

Finally, information on B. maritima usually has been abbreviated into a short chapter or part of chapter in books on sugar beet breeding and genetics, but has never received comprehensive coverage. For these reasons, the book will serve as a valued reference for students, researchers, scientists, molecular biologists, and breeders.
P. Stevanato $(\square)$

DAFNAE, University of Padova, Agripolis, Viale

dell'Università, 16, 35020 Legnaro, PD, Italy

e-mail: stevanato@unipd.it

URL: http://scholar.google.it/citations?user=Gc9S8ucAAAAJ 